\title{
Contribution of immunophenotype in the diagnosis and classification of haemopoietic malignancies
}

\author{
E Matutes
}

\section{Introduction}

There have been major advances over the past decade in the immunophenotypic characterisation of haemopoietic malignancies. This is a consequence of the development of the hybridoma technique by Kohler and Milstein in the mid-1970s ${ }^{1}$ and the subsequent production of monoclonal antibodies which detect specific antigenic determinants on cells from the various haemopoietic lines and are often restricted to particular stages of differentiation or maturation.

Before the availability of monoclonal antibodies, the standard methods for characterising acute leukaemias and lymphoproliferative disorders utilised polyclonal antibodies against human immunoglobulins, class II HLA DR determinants, and the common acute lymphoblastic leukaemia (ALL) antigen (c-ALLA), expressed in lymphoblasts from most cases of ALL, rosetting tests, such as sheep erythrocytes rosettes (E-rosettes), and a biochemical assay to detect the nuclear enzyme terminal deoxynucleotidyl transferase (TdT).

These tests permitted the classification of most cases of ALL into two cell types: B cell lineage (TdT +, c-ALLA +, HLA DR + , and E-rosette negative) and $T$ cell lineage (E-rosette positive, TdT + , c-ALLA - , HLA DR -) and distinguished ALL (TdT + ) from the chronic lymphoproliferative disorders of mature $B$ and $T$ cells $(\mathrm{TdT}-){ }^{2}$ In addition, the more mature type of B cell derived ALL or Burkitt's lymphoma (L3-ALL) was characterised by the expression of membrane immunoglobulin and was usually TdT and c-ALLA negative. At this time a subset of ALL was designated null ALL, in which blasts were TdT + , HLA DR + but were c-ALLA and E-rosette negative. ${ }^{2}$ The latter subgroup, with the use of $B$ cell lineage specific monoclonal antibodies, has now been recognised as B cell precursor ALL (see later). The diagnosis of acute myeloid leukaemia (AML) was, at this time, largely based on cell morphology and cytochemistry and on negative findings on staining with antilymphocyte antibodies.

Because of the great number and variable specificity of available reagents, it soon became apparent that there was a need to standardise the nomenclature of antibodies reactive against specific leucocyte differentiation antigens. The various monoclonal antibodies which recognise different epitopes of a particular antigen are now given a cluster of differentiation (CD) number. Over $100 \mathrm{CD}$ numbers have been assigned so far and each CD comprises a number of monoclonal antibodies. ${ }^{3}$ There have also been major advances in the technology used to detect the expression of antigens on leukaemic and normal cells. This is reflected by (a) detection of both membrane and cytoplasmic antigens, not only by immunocytochemical techniques such as the immunoalkaline phosphatase-antialkaline phosphatase (APAAP) method on fixed cytocentrifuge slides, but also by flow cytometry; (b) the ability to stain for more than one antigen using directly labelled monoclonal antibodies with different fluorochromes; and (c) the ability to quantify the number of molecules expressed on a particular cell, which may have important diagnostic implications, or for the detection of minimal residual disease.

Despite the large number of monoclonal antibodies available, only a few are useful for diagnosis. Other markers still in use include Ki67, which measures the proliferative rate of a tumour, and those directed against the multidrug resistance glycoproteins, which may be of prognostic relevance.

\section{Acute leukaemias}

The nature of the neoplastic cells in most cases of acute leukaemia can now be established by applying a small battery of monoclonal antibodies which detect differentiation antigens expressed in early lymphoid and myeloid cells (fig 1). Immunophenotyping is essential to characterise the various subtypes of ALL and also certain types of AML, in which morphology and cytochemistry fail to disclose the origin of the blasts such as those with poorly differentiated myeloblasts (M0-AML) ${ }^{4}$ and AML derived from early erythroid (M6-AML) and megakaryoblastic cells (M7-AML) $)^{5-8}$ Techniques that facilitate the detection of some of the antigens in the cytoplasm are very useful because the most specific markers for the lymphoid and myeloid cell lineages are only expressed in the cell membrane at late stages of differentiation but are positive early on in the cytoplasm. ${ }^{6-8}$

An important aspect to consider is the specificity of the markers. Whilst some of them are highly sensitive and specific for a particular cell line, such as $\mathrm{CD} 3$ for $\mathrm{T}$ lymphoblasts, mb-1 (CD79a) for B lymphoblasts and antimyeloperoxidase (anti-MPO) for myeloid cells, other markers-for example, CD2, CD10 and $C D 13$, are less specific. Nevertheless, they 


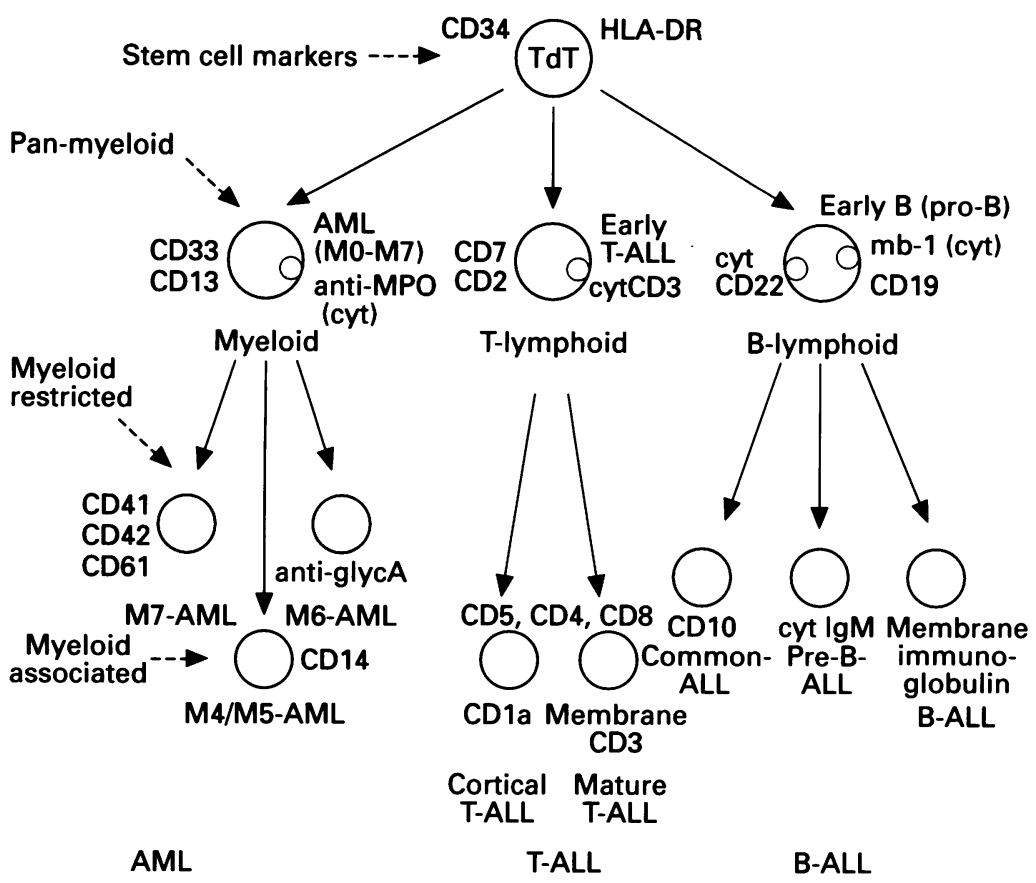

Figure 1 Immunological markers in the classification of acute leukaemias.

are also valuable for determining whether the blasts are committed to lymphoid or myeloid pathways and/or when findings are negative with the most specific markers. Another set of markers are those directed against stem cell precursors, such as TdT and CD34, which can also be useful in distinguishing mature and immature lymphoid malignancies. CD34 may also have prognostic relevance.

\section{ACUTE LYMPHOBLASTIC LEUKAEMIAS}

$B$ and $T$ cell derived ALL can be further classified into various subgroups based on the expression of lymphoid differentiation antigens (fig 1). Although not essential this is important because of the association between some ALL subtypes and specific cytogenetic findings or clinical outcome-for example, pre-B-ALL and the $t(1 ; 19)$ translocation. Four types of B cell ALL (TdT +, CD19+, mb-1 +, and cytCD22 +) can be distinguished using markers: early $B$ cell precursor (pro-B-ALL) $(\mathrm{CD} 10-)$, previously designated null-ALL, common-ALL (CD10+), pre-B-ALL (cytoplasmic $\mu+$ ), and mature B-ALL (surface immunoglobulin +) (fig 1). The subclassification of $\mathrm{T}$ cell derived ALL (TdT+, cytCD3 + and CD7 +) has not been well defined as yet. On the basis of normal thymic differentiation, at least three subgroups can be distinguished: early and pre-T-ALL (CD7+, cytCD $3+$ and $\mathrm{CD} 2+/-)$, cortical T-ALL $(\mathrm{CDla}+)$ and mature T-ALL (membrane CD3 +) (fig 1).

\section{ACUTE MYELOID LEUKAEMIAS}

In AML (CD33 $+/-, \mathrm{CD} 13+/-$ and anti$\mathrm{MPO}+/-)$ there are no myeloid markers which enable the AML subtypes M1 to M5 of the French-American-British (FAB) classification to be distinguished. However, some markers may be preferentially expressed in some types, such as CD14 when there is evidence of monocytic differentiation (M4 and M5) or negative class II HLA DR cases with marked myeloid differentiation such as M3-AML or late M2-AML. The M2-AML phenotype is also characteristic as most cases express the $B$ lymphoid marker CD19. There are also some markers which are highly specific for certain myeloid lines and thus useful for characterising erythroid and megakaryoblastic acute leukaemias. These are monoclonal antibodies against glycophorin $\mathbf{A}$ for the erythroid cells and three monoclonal antibodies, CD41, CD42 and CD61, which recognise the glycoproteins Ib, the IIb/IIIa complex and IIIa in the membrane of the platelets and megakaryocytes (fig1).

\section{ACUTE BIPHENOTYPIC LEUKAEMIAS}

Immunological marker analysis has facilitated the recognition of unusual (about $8 \%$ ) cases of acute leukaemia of biphenotypic or mixed cell lineage, in which cells coexpress markers characteristic of myeloid and lymphoid lineages and which may represent leukaemias derived from stem cells. There is no agreement on the nomenclature and definition of biphenotypic acute leukaemias. The lack of uniform criteria defining this particular acute leukaemia subset hinders our ability to determine whether or not it constitutes a distinct clinical entity with prognostic implications. In this context we have proposed a scoring system which takes into account the specificity and the number of markers expressed in the neoplastic cells to aid differentiation of the truly biphenotypic from those with aberrant expression of a marker from another lineage. ${ }^{910}$

\section{Chronic or mature lymphoproliferative} disorders

Immunophenotyping in chronic or mature lymphoproliferative disorders has permitted more precise characterisation of the neoplastic cells, leading to the recognition of well defined disease entities. ${ }^{11}$ Immunological markers play a key diagnostic role by enabling demonstration of the B or T cell nature of the neoplastic cells; by establishing clonality in B cell disorders by immunoglobulin light chain restriction analysis and thus, distinguishing neoplastic from reactive B lymphocytosis; and by distinguishing immature or lymphoblastic malignancies $(\mathrm{TdT}+$ ) from neoplasms of mature lymphoid cells $(\mathrm{TdT}-)$. There are also particular characteristic phenotypic profiles in some of these disorders which are useful for diagnostic purposes. Examples of these are the marker profiles in chronic lymphocytic leukaemia (CLL) and hairy cell leukaemia (HCL). Cells from most cases of CLL are CD5+ and CD23+, FMC7 - and weakly express immunoglobulin and CD22 in the membrane, or are negative for the latter (fig 2). Because there is no single 


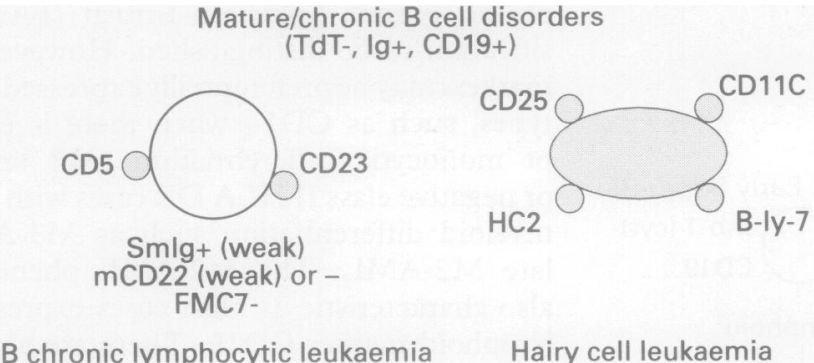

Mature T cell disorders (TdT-, CD1a-)
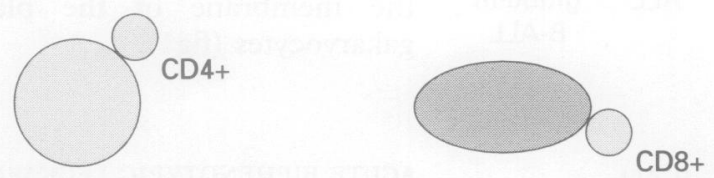

T prolymphocytic leukaemia (T-PLL) Adult T cell leukaemia lymphoma (ATLL) Sézary's syndrome (SS)

Peripheral T cell lymphomas

Large granular lymphocyte (LGL) leukaemia

\section{Associated $\longrightarrow \mathrm{CD} 7+(\mathrm{T}-\mathrm{PLL}) \longrightarrow \mathrm{NK}$ markers markers $\longrightarrow$ CD25+ (ATLL) $\longrightarrow$ (CD11b, CD16, CD56, CD57)}

Figure 2 Characteristic immunological profiles in chronic lymphoproliferative disorders. NK $=$ natural killer. normal peripheral blood T lymphocytes, such as the lack of reactivity with pan-T reagents (for example, CD2), or coexpression of CD4 and natural killer associated markers (for example, CD56 or CD11b). Such immunophenotypes may be helpful by indicating the clonal/neoplastic nature of the $T$ cells, as there is no immunological marker that permits the demonstration of $T$ cell clonality. These marker profiles can also be used to detect minimal residual disease. Marker profiles clearly overlap in the various $\mathrm{T}$ cell disorders. Most of them, T prolymphocytic leukaemia (T-PLL), Sézary's syndrome and adult $\mathrm{T}$ cell leukaemia lymphoma (ATLL) correspond to expansions of CD4 +, CD8 - cells whereas the phenotype in large granular lymphocyte (LGL) leukaemia is often $\mathrm{CD} 8+, \mathrm{CD} 4-.{ }^{15}$ Although there is no single marker specific to a disease entity, it seems that certain markers are more characteristic of some diseases, such as the strong expression of receptors for the alpha chain of interleukin-2 (CD25) in ATLL, natural killer associated markers in LGL leukaemia or strong reactivity with CD7 in T-PLL (fig 2). ${ }^{15}$

In the context of lymphoid disorders, markers also play a major role in disclosing the lymphoid nature of the neoplastic cells in cases of large cell lymphoma which present with clinical and cytological features resembling AML. ${ }^{16}$

In summary, the systematic use of immunological markers over the last two decades has permitted better and more precise characterisation and classification of haemopoietic malignancies leading to the recognition of well defined disease entities. This, in turn, has significantly improved our understanding of normal haemopoiesis and has given new insights into the pathogenesis of some of these neoplasms. system which considers the pattern of expression of these five markers. We have shown that this scoring system is valuable in distinguishing CLL, including cases with atypical morphology, from other B cell disorders. ${ }^{12}$

The immunological profile of $B$ cell malignancies other than CLL may be very similar. However, there are some monoclonal antibodies which are valuable for distinguishing between three disorders with circulating villous or hairy lymphocytes, namely typical HCL, the variant form of $\mathrm{HCL}$ (HCL-variant) and a low grade lymphoma known as splenic lymphoma with villous lymphocytes (SLVL). In these conditions the pattern of reactivity with four monoclonal antibodies, which had been considered HCL specific, namely CD11c, CD25, HC2, and $\mathrm{B}-\mathrm{ly}-7$, is different. Although no marker is unique to $\mathrm{HCL}$, as they may be positive in the HCL-variant and SLVL cells, typical HCL cases express at least three of the four markers whereas cases of HCL-variant or SLVL express no more than two of them. ${ }^{1314}$

When considering mature or chronic $T$ lymphoproliferative disorders, markers are useful not only for establishing the $T$ cell origin of the neoplastic cells but also for distinguishing them from lymphoblastic malignancies by the differential expression of TdT and CD1a. Immunophenotyping may also disclose aberrant or atypical phenotypes usually not found in

Kohler G, Milstein C. Continuous cultures of fused cells secreting antibody of predefined specificity. Nature 1975; 256:495-7.

2 Greaves MF, Janossy J, Peto J, Kay H. Immunologically defined subclasses of acute lymphoblastic leukaemia in children: their relationship to presentation features and prognosis. Br f Haematol 1991;48:179-97.

3 Tissue Antigens. 5th International Conference on Human leukocyte differentiation antigens. Vol. 42. Munksgaard Copenhagen, 1993:165-464.

4 Bennett JM, Catovsky D, Daniel MT, Flandrin G, Galton DAG, Gralnick HR, et al. Proposal for the recognition of minimally differentiated acute myeloid leukaemia. $\mathrm{Br} f$ Haematol 1991;78:325-9.

5 Chan LC, Pegram SM, Greaves MF. Contribution of immunophenotype to the classification and differential diagnosis of acute leukaemia. Lancet 1985; i:475-9.

6 Janossy G, Coustan-Smith E, Campana D. The reliability of cytoplasmic CD 3 and CD22 antigen expression in the immunodiagnosis of acute leukemia - a study of 500 cases. Leukemia 1989;3:170-81.

7 Buccheri V, Shetty V, Yoshida N, Morilla R, Matutes E, Catovsky $\mathrm{D}$. The role of an anti-myeloperoxidase antibody in the diagnosis and classification of acute leukaemia: a comparison with light and electron microscopy cytochemistry. Br f Haematol 1992;80:62-8.

8 Buccheri V, Mihaljevic B, Matutes E, Dyer MJS, Mason DY, Catovsky D. mb-1: a new marker for B-lineage lymphoblastic leukemia. Blood 1993;82:853-7.

9 Matutes E, Buccheri V, Morilla R, Shetty V, Dyer M Catovsky D. Immunological, ultrastructural and molecular features of unclassifiable acute leukaemia. In: $R \boldsymbol{e}-$ cent results in cancer research. Vol 131. Berlin: SpringerVerlag, 1993:41-52.

10 Catovsky D, Matutes E, Buccheri V, Shetty V, Hanslip J, Yoshida $\mathrm{N}$, et al. A classification of acute leukaemia for the 1990s. Ann Haematol 1991;62:16-21.

11 Matutes E, Catovsky D. The classification of lymphoid leukaemias. Leuk Lymphoma 1991;5(Suppl):153-5.

2 Matutes E, Owusu-Ankomah K, Morilla R, Garcia-Marco J, Houlihan A, Que L, et al. The immunological profile 
of B-cell disorders and proposal of a scoring system for the diagnosis of CLL. Leukemia 1994;8:1640-5.

13 Matutes E, Morilla R, Owusu-Ankomah K, Houlihan A, Meeus P, Catovsky D. The immunophenotype of hairy cell leukemia (HCL). Proposal for a scoring system to distinguish HCL from other B-cell disorders with hairy or villous lymphocytes. Leuk Lymphoma 1994;14(Suppl 1):57-62.

14 Matutes $E$, Morilla $R$, Owusu-Ankomah $K$, Houlihan $A$, Catovsky D. The immunophenotype of splenic lymphoma with villous lymphocytes and its relevance to the differential diagnosis with other B-cell disorders. Blood 1994 83:1558-62.

15 Matutes E, Catovsky D. Mature T-cell leukaemias and leukaemia/lymphoma syndromes: review of our experience in 175 cases. Leuk Lymphoma 1991;4:81-91.

16 Bain B, Matutes E, Robinson D, Lampert IA, BritoBabapulle V, Morilla $\mathrm{R}$, et al. Leukaemia as a mani-
festation of large cell lymphoma. Br $\mathcal{f}$ Haematol 1991; 77:301-10. 